\section{AN ESSAY IN CORRELATION ARISING FROM ALKALOID CHEMISTRY*}

\section{BY SIR ROBERT ROBINSON, F.R.S.}

$\mathrm{T}$ HE incident which I wish to describe is of peculiar interest, because it transpires that the behaviour of a natural product can only be explained in the light of knowledge comparatively recently acquired and never previously brought to bear in the analysis of a structural problem.

When we break up molecules in order to examine the fragments, or submit them to transformations in order to recognize the reactive groups, the changes that occur usually follow relatively simple rules; the pieces, for example, are clearly related to the whole from which they were obtained. In certain classical instances the picture has been confused by rearrangements of atoms within the molecule in the course of some of the changes examined.

But these molecular shifts also follow laws which have gradually been brought to light, and it has always been possible to integrate the data in terms of a particular molecular structure.

Thus camphor showed itself to be a chemical proteus and at one stage it was not clear which manifestation corresponded to the reality. The vagaries of this molecule which proved most puzzling were explained by Lapworth. Camphor and all its derivatives have been synthesized and thus all dubiety was resolved.

Brazilin is another molecule which twists itself into contortions, and here again we can follow all the varied transformations, and we have the confirmation of synthesis, not, it is true, of brazilin itself, but of many of its more important derivatives.

But the star performers in the team of molecular acrobats are undoubtedly the alkaloids of the morphine group, and I shall speak especially of thebaine.

In 1923-25 I discussed the chemistry of morphine, codeine and thebaine with John Masson Gulland, whose untimely death in a recent railway accident was so great a tragedy. We arrived at a solution of the problem which has since been generally accepted, but there is no synthetic proof that applies to the thebaine structure itself. The certain knowledge from analysis and synthesis relates to the various transformation products, and the molecular structures of many of these are established beyond doubt. The validity of our formulæ, therefore, is estimated entirely on their success in interpretation of a complex of data. With an emendation in one group of products, suggested by Schöpf and gladly accepted, it seemed that the book was all but closed. Yet the most remarkable rearrangement of the molecule and one of the most extraordinary changes yet encountered in the whole domain of organic chemistry remained to be recognized. It subjected the Gulland-Robinson formula to a very severe test, and its triumphant emergence from the ordeal was at one time in doubt and has only been guaranteed during the last two months.

In 1905 Freund treated thebaine with phenylmagnesium bromide and obtained a new base, phenyldihydrothebaine, which was just thebaine to which the elements of benzene $\left(\mathrm{C}_{6} \mathrm{H}_{5}, \mathrm{H}\right)$ had been added. Similar substances were made later using

* From the presidential address to the Royal Society, delivered on December 1. other organo-metallic reagents, and Lyndon Small and his collaborators have carefully re-examined and extended the series. They have established some intriguing stereochemical relationships and corrected mistakes of other workers.

In 1943 , in the course of a visit to Washington, I was privileged to hear of Dr. Small's results and to examine the detail of his brilliant experimental work. Two conclusions appeared to me then to be inescapable; but the full solution of the mystery remained elusive. In September of this year Dr. Small kindly sent me the manuscript of a paper intended for publication in the Journal of Organic Chemistry. He had been unable to make any use of my views, and further correspondence showed why that was so, and also led to the understanding that the present disclosure should be made. A new idea, based on the postulates of 1943, then occurred, and the validity of this has recently been indicated by a crucial experiment.

The first of the inescapable conclusions to which I have referred is derived from a comparison of thebaine with phenyldihydrothebaine. The latter is outstandingly more stable. It is hard to hydrogenate in the presence of a catalyst (thebaine is easily attacked), and when reduction does occur all that happens is a break of a $\mathrm{C}-\mathrm{N}$ bond. Thebaine is very easily hydrolysed as an enol methyl ether; but although phenyldihydrothebaine contains this methoxy group (also one other $\mathrm{OCH}_{3}$ and a phenolic $\mathrm{OH}$ ), it is not hydrolysed by hot concentrated hydrochloric acid. But hot hydrobromic acid produces the change $\left(\mathrm{OCH}_{3}\right)_{2}(\mathrm{OH}) \rightarrow(\mathrm{OH})_{3}$, and the action of diazomethane on the trihydroxy-compound gives a trimethoxy-compound which is the methyl ether of phenyldihydrothebaine. From these facts it is perfectly clear that the near-aromatic nucleus of thebaine, which bears the hydrolysable methoxyl, has become truly aromatic in phenyldihydrothebaine.

The second conclusion follows logically from the first, considered in the light of other data. Phenyldihydrothebaine is optically active and by Hofmann's exhaustive methylation procedure it gives a series of optically active derivatives; this is even true of the second stage in which nitrogen is eliminated. The process is $\mathrm{C}-\mathrm{C}-\mathrm{N}-\mathrm{C}-\mathrm{C} \longrightarrow \mathrm{C}=\mathrm{C}: \mathrm{NM}_{3} \vdots \mathrm{C}=\mathrm{C}$. The two double bonds in this optically active product can easily be shown to be present by diagnostic tests.

Now phenyldihydrothebaine is $\mathrm{a}_{25} \mathrm{C}_{25}$ base and one carbon atom is in : $\mathrm{NCH}_{3}$ (now lost), two carbon atoms are in $\mathrm{OCH}_{3}$ groups, and by hypothesis eighteen carbon atoms are in three benzene rings (two of the thebaine skeleton and the phenyl group introduced by the reagent); that leaves only four carbon atoms for the two double bonds. Hence exhaustively methylated phenyldihydrothebaine cannot contain an asymmetric carbon atom and it must owe its optical activity to an asymmetric molecule of the type familiar to synthetic chemists and stereochemists but never before encountered in the study of a natural product.

It is easy to guess what kind of an asymmetric molecule is present. Thebaine is easily degraded to phenanthrene derivatives (such as I) and these in their turn are derived from diphenyl (II).

In 1922 Christie and Kenner showed that the dinitrodiphenic acid (III) could be resolved into optically active forms. They also pointed out that this result was explicable if the two rings are assumed to be co-axial (as shown by the dotted line) but not co-planar. In 1926 Turner and Le Fèvre, Bell and 


\section{6}<smiles></smiles>

(I)

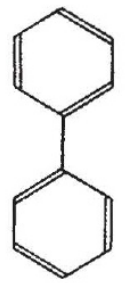

(II)

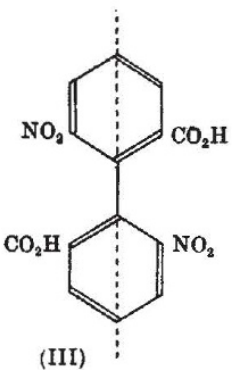

(III)
Kenyon, and Mills developed the hypothesis that restricted free rotation of the phenyl groups is conditioned by the position and size of the substituents. It is well known that this hindrance theory harmonizes all the extensive experimental data.

We arrived at the restricted diphenyl idea as a plausible explanation of the optical activity of the end-product of the Hofmann degradation; but there is strong evidence that it must also be applied to phenyldihydrothebaine itself.

Small and his collaborators have shown that Freund's phenyldihydrothebaine is a mixture of two isomerides (equated in various ways, as by reduction, or exhaustive methylation) which he calls $(+) \alpha$ and $(+) \delta$. On heating, $(+) \alpha$ is partly changed to the optical antipode of $(+) \delta$; similarly $(+) \delta$ goes partly into the optical antipode of $(+) \alpha$. The equilibria, reached from either side, favour the $\alpha$ forms. Small clearly recognizes that these changes are partial racemizations but appears to think it possible that a number of asymmetric centres could be inverted in step. He calls the optical antipode of $(+) \alpha,(-) \alpha$; but it is preferable to term it $(-) \delta$. Thus + , - and $\alpha, \delta$ can be taken to be symbols representing left-hand or right-hand at each of two sources of dissymmetry. The partial racemization of $(+) \alpha$ gives then $(-) \alpha$. These facts alone suggest that the phenyldihydrothebaine molecule contains two sources of dissymmetry and only two; one of these (represented by,+- ) is a non-co-planar diphenyl configuration, and the other (represented by $\alpha, \delta$ ) is an asymmetric carbon atom. We are able to substantiate this view, and the substance is therefore the first in which molecular dissymmetry, and that due to an asymmetric carbon atom (also, strictly speaking, molecular), co-exist. The theory extends to this case without modification; there are the usual numbers of isomerides, $2^{n}$, where $n$ is the number of sources of dissymmetry, not necessarily all asymmetric carbon atoms. This prinoiple is quite obvious but has not previously been enunciated, chiefly because the mixture of asymmetric types is itself new.

All these and many other facts had to be taken into consideration in the endeavour to find a structure for phenyldihydrothebaine, and it would take too long

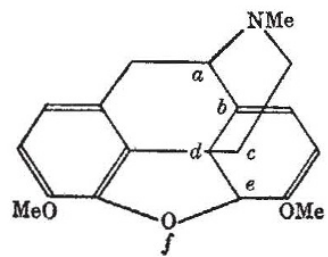

(IV)

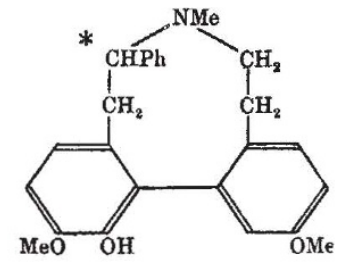

(V)

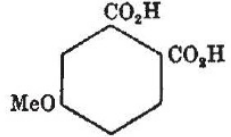

(VI) to carry the argument through all its stages. It will be stated more fully elsewhere; meanwhile phenyldihydrothebaine is believed to be (V) and its formation from thebaine (IV) is the result of molecular rearrangement of quite a new type.

It was first formulated as a likely process from the electronic point of view. In words: the migrating ethanamine chain is cationoid, $(c)$ is joined to $(b)$ by means of the electrons of the link $(a b)$; the electrons in $(c d)$ go to $(d e)$ and fulfil the demands of aromaticity of the nucleus; electrons $(e f)$ are then taken by the oxygen atom which acquires a negative charge (on decomposition of the complex by water, a proton is attached to oxygen); the phenyl anion brings a new migrating group (c). This connected series of changes can be thought of as starting at either end ; namely, attack by $\mathrm{Ph}-$, or assumption of a negative charge by oxygen. As in many other migrations in this group, the driving force is doubtless the tendency of the near-aromatic group to become fully aromatic. The formation of isomerides is due to construction of the -CHPh- group in each of the two possible senses, the diphenyl skeletons being configured in one sense only.

The formula (V) is perfectly satisfactory; examination of models shows that the nine-membered ring allows the phenyl nuclei to be disposed at right angles or at any intermediate angle without strain; there is one asymmetric carbon atom marked * in the formula (V); all the chemical properties and transformations receive natural explanations. But there was thought to be one important exception, time before the complete theory was available) to accept the postulation of the two aromatic nuclei. The ultra-violet absorption of phenyldihydrothebaine gave no indication whatever of the appearance of a new aromatic ring in conjugation with the first. This ought to have been thought decisive, but as it conflicted with other equally definite evidence, something had to be jettisoned. Actually a further correlation is possible, and the parts now fit together like a Chinese puzzle made in ivory.

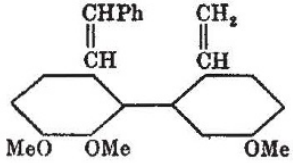

(VII)

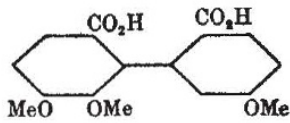

(VIII) electron pair to $(a)$, replacing those lost to the and because of it Dr. Small was not disposed (some

I am indebted to Dr. J. W. Cornforth for pointing out that non-co-planar diphenyls do not exhibit normal ultra-violet spectra. This was first noted by Picket, Walter and France (1936), and a clear case is given by O'Shaughnessy and Rodebush (1940). In this example a large ring of eighteen members is formed by a bridge across the $m$-positions of a diphenyl derivative. There is no diphenyl band and only end-absorption.

In the last few weeks we have sought and obtained experimental confirmation of the structure (V). Mr. $\mathrm{K}$. W. Bentley has oxidized phenyldihydrothebaine with potassium permanganate and obtained benzoic acid, benzaldehyde, and 4-methoxyphthalic acid (VI). This was identified very thoroughly with an authentic specimen.

By exhaustive methylation, including that of the phenolic group, an optically active product (VII) 
(made by Freund but stated to be optically inactive) was obtained, and oxidation of this by permanganate in acetone afforded a trimethoxydiphenic acid (VIII). These are very cold, hard facts, and it is to be feared that in the future they will be cited first and all our interesting arguments will be forgotten.

One further punctilio. The thebaine transformations are like the variations of a chess problem in that they have a common key-move. Are we justified. in assuming that the problem as set has a unique relation to the variations ? Is the thebaine structure proposed by Gulland and Robinson the only one which serves to explain the whole of the results? The key-move is the migration of the ethanamine chain from $(d)$ to $(b)$ in (IV) ; it is only the position after the migration that we have proved to be correct. Taking everything into consideration, one alternative can indeed be seen. This can best be explained by reference to the intermediate (IX) now postulated in the complex changes that thebaine undergoes in acid solution.

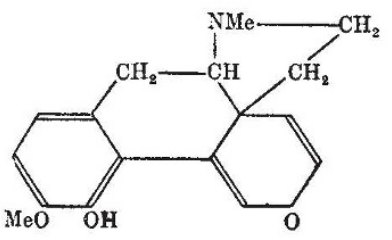

(IX)

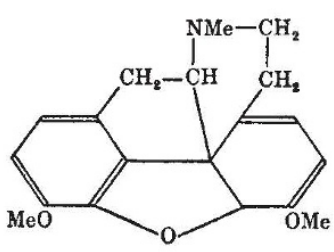

(X)
(IX) arises from (IV) by migration from $(b)$ to $(d)$ and by hydrolysis. It could equally well arise from (X). The only way of distinguishing between (IV) and (X) seems to be by a study of the $\alpha$-and $\beta$-methylmorphimethines; and the optical data on these substances strongly support (IV) and would remain inexplicable on the basis of (X). According to Dr. $R$. Strauss, the ultra-violet absorption of $\alpha$-methylmorphimethine is not styrenoid and would be consistent with the arrangement, $\varphi . C . C: C . C: C-$. On the other hand, that of the $\beta$-isomeride shows strong conjugation consistent with $\varphi . C: C . C: C$. . The skeleton of (IV) permits these rearrangements and structural elements, but that of $(\mathrm{X})$ does not. The question is related to that of biogenesis and further inquiries must be instituted.

\section{THE INTERNATIONAL METEOR- OLOGICAL ORGANISATION}

\section{MEETINGS IN TORONTO AND WASHINGTON}

$\mathrm{T}$ HE meetings of the International Meteorological Organisation which were held in Toronto and Washington between the beginning of August and mid-October 1947 were of unusual interest, not only on account of the results achieved, but also on account of some of the precedents that were created. It was the first time in its seventy-five years history that any of the constituent bodies of the Organisation had met in the New World, and it was also the first occasion that all ten of the technical oommissions had gathered together concurrently.

During the War, the activities of the Organisation were seriously curtailed, but as soon as possible after the return of peace, steps were taken to set its machinery in motion once again. An Extraordinary Conference of Directors took place in London during February 1946; new members of the International Meteorological Committee were elected and the technical and regional commissions were reconstituted. This Conference thus cleared the way for a resumption of the international procedures in operation before the War and for the world-wide introduction of improvements in the field of scientific meteorology made by certain countries during the War.

The objects of the meeting at Toronto and Washington were therefore to review and consolidate the progress made since the London conference, to make plans for the future and to consider the structure of the International Meteorological Organisation and its relationship to other international associations.

The meetings of the ten technical commissions in Toronto were spread over six weeks in August and September, and were attended by 179 delegates from forty-four countries. The scope of the procoedings was so wide and the discussions so detailed that even a summary of the activities of each commission would be too lengthy, and attention must therefore be restricted to an arbitrary selection of the main items.

The Aeronautical Commission, which was in session for three weeks, undertook the preparation of a common text for regulations to be adopted by both the International Meteorological Organisation and the International Civil Aviation Organisation for the provision of meteorological facilities to aviation. Codes for the transmission of weather reports to and from aircraft, and for the transmission of terminal and route forecasts between ground stations and to aircraft in flight, required lengthy discussion before agreement was obtained. The Commission for Maritime Meteorology dealt with such problems as marine climatology, the reporting of sea ice by ships, the measurement of ocean waves and swell, and means of increasing the frequency and accuracy of weather reports received from ships. The operation of ocean weather ships and their most suitable stations were also discussed.

The construction of a basic code for weather observations, with specifications for the various elements to be reported, was one of the chief tasks of the Commission for Synoptic Weather Information, and a code acceptable to all countries was eventually devised. Other subjects considered included methods of representing upper-air data on charts, the standard times of synoptic reports and the arrangements for the collection and distribution of meteorological information. The Commission for Instruments and Methods of Observation was formed at the London Conference in 1946, and met for the first time at Toronto, although in the intervening period much work had been done by correspondence. A comprehensive agenda had been prepared, and the Commission surveyed the whole field of meteorological instruments. The discussions usually turned on questions of standardization; but it was generally felt that only a limited degree of standardization should be the present objective, because much of the equipment in current use was relatively new and capable of further development. It was agreed, however, that comparison of different types of radio-sonde is necessary, and recommendations were made for the design of a simple reference instrument. Problems of visibility measurement were also dis- 